\title{
Vom liberalen zum konservativen judicial »activism«? Die Entwicklung der amerikanischen Verfassungsrechtsprechung in den letzten fünfzig Jahren
}

\section{Einleitung}

Als die Richterin Sandra Day O'Connor am 1. Juli dieses Jahres ihren Rücktritt vom Supreme Court bekannt gab, war das Erstaunen innerhalb der politischen Elite Washingtons und an ihrer politischen Basis groß. War vor zwei Jahren noch heftig über eine bevorstehende Abdankung der obersten Richterin spekuliert worden, so richtete sich die allgemeine Erwartung Ende Juni darauf, dass der schwerkranke Chief Justice des Gerichts William H. Rehnquist seinen Hut nehmen würde ${ }^{1}$. Der dementiert jedoch weiterhin jegliche Rücktrittsgerüchte und löst damit wiederum Spekulationen über seine Beweggründe für das starre Festhalten am Amt aus².

Washington steht also im wahrsten Sinne des Wortes ein »heißer Sommer «evor, denn anders als in Deutschland, wo die Ernennung von Verfassungsrichtern hinter verschlossenen Türen zwischen den Parteien ausgehandelt wird, ist der Nominierungsprozess in Amerika ein öffentliches und hoch politisches "Spektakel«, an dem neben dem Präsidenten und seinen Beratern die Senatoren und eine Vielzahl von konservativen und liberalen Lobbygruppen beteiligt sind. Vor allem bei letzteren ist der Mobilisierungsprozess bereits im vollem Gang - und dies obwohl der Präsident seine Kandidatenwahl noch gar nicht bekannt gegeben hat. Schätzungen ergeben, dass bei dem Kampf um die Ernennung eines Nachfolgers für Frau O'Connor diesen Sommer die Lobbygruppen bis zu 50 Millionen Dollar ausgeben werden. Warum löst die Neubesetzung einer Richterstelle am Supreme Court eine so massive politische Mobilisierung aus?

Die zurückliegenden Sitzungsperioden des Obersten Bundesgerichtshofs verdeutlichten einmal mehr den politischen Gestaltungsspielraum der dritten Gewalt in den Vereinigten Staaten. So wurden innenpolitische Kontroversen über verfassungsrechtliche Themen in den letzten fünf Jahrzehnten weniger durch die Legislative, sondern fast ausschließlich vor dem Supreme Court entschieden. Das hohe

1 Douglas Jehl, »At White House, Surprise Is Borne Out in Name Only« in: The New York Times, 02.07.2005.

2 Linda Greenhouse, » Rehnquist Denies Rumor of Retirement« in: The New York Times, 15. 07. 2005. 
Quorum, das nötig ist, um die Verfassung zu ändern, und die Befugnis des judicial review haben diese Entwicklung maßgeblich bedingt ${ }^{3}$.

Am 15. Januar 2003 erklärte der amerikanische Präsident George W. Bush in einer Rede, dass seine Regierung eine vor dem Supreme Court anhängige Klage dreier weißer StudentInnen unterstützen werde, die sich gegen die Quotenregelung der Universität von Michigan richtete. Anhand dieser Quotenregelung wurden im Rahmen der affirmative action beim Zulassungsverfahren der Universität Zusatzpunkte an Angehörige von Minderheiten vergeben. Befürworter der Politik der Minoritätenförderung kritisierten den keineswegs unerwarteten Vorstoß der Bush-Administration. Sie sehen den Zeitpunkt eines Förderungsstopps noch nicht gekommen, zumal eine bessere Alternative, den im amerikanischen Selbstverständnis verankerten Grundsatz der »equal opportunities « zu realisieren noch nicht gefunden scheint. Zudem wurde der gewählte Zeitpunkt des Vorstoßes der Bush Regierung kritisiert, der nur wenige Tage vor dem Martin Luther King Day am 20. Januar 2003 erfolgte.

Außerdem sieht sich Präsident Bush mit Forderungen der religiösen Rechten konfrontiert seine Wahlkampfversprechen vom Jahr 2000 einzulösen, besonders was die Abschaffung der Möglichkeit legaler Abtreibung anbelangtt. Angesichts des 30. Jahrestages der Entscheidung Roe v. Wade (1973) vor dem Supreme Court der Vereinigten Staaten wurden am 22. Januar 2003 die Stimmen bezüglich der »notwendigen « Revision dieses Urteils wieder lauter ${ }^{5}$. Die Erwartung vieler Abtreibungsgegner geht dahin, dass, sobald die nächste vakante Richterstelle durch Präsident Bush neu besetzt wäre, eine Revision des Präzedenzfalles von 1973 in greifbarer Nähe ist. Dies setzt jedoch voraus, Präsident Bushs Wahl träfe tatsächlich auf einen ultrakonservativen Richter, der den Vorstellungen der religiösen Rechten entspricht.

Allein diese zwei innerhalb der Vereinigten Staaten kontrovers diskutierten Themen zeigen die eminente Bedeutung des Supreme Court im politischen Prozess des Landes auf und verdeutlichen die Brisanz der Besetzung der höchstrichterlichen Positionen und deren mögliche Auswirkungen auf eine Vielzahl besonders umstrittener Verfassungsfragen. Noch nie war die judicial branch in der über 200 Jahre alten Geschichte des politischen Systems der Vereinigten Staaten so einflussreich und "politisch« aktiv, wie in den vergangenen fünf Jahrzehnten. Dabei ist der Supreme Court der Vereinigten Staaten mehr als jedes andere Verfassungsgericht dazu gezwungen, die politischen Entscheidungen, die er zwangsläufig treffen muss, zu rechtfertigen. Von ihm wird erwartet, dass er sich stets als unpolitische, rein judika-

3 Um die Verfassung in den USA durch ein amendment zu ergänzen bedarf es außer der 2/3 Mehrheit in beiden Häusern des Kongresses auch der Ratifizierung durch 3/4 der Bundesstaaten.

Das judicial review, die richterliche Befugnis, Gesetze und Rechtsverordnungen auf ihre Verfassungsmäßigkeit zu überprüfen ist in den USA auf konkrete Fälle beschränkt. Die Kompetenz der abstrakten Normenkontrolle wie das Bundesverfassungsgericht sie besitzt, hat der Supreme Court nicht.

4 »Republican Party 2000 Convention Extra. Bushville, U.S.A.« in: Newsweek, Vol. 136, 7. 8. 2000, S. 30; Dean McSweeney, "Political Parties « in: Gillian et al. (Hg.), Developments in American Politics 4, Houndmills u. a. 2002, S. 35-52, S. 44.

5 Debra Rosenberg, »In Roe's Shadow « in: Newsweek, B 141/2003, S. 58-59. 
tive Gewalt darstellt. Andernfalls läuft er Gefahr, seine Autorität und Legitimation bei der amerikanischen Bevölkerung einzubüßen. Doch gerade weil die Hürden für eine Verfassungsänderung in den Vereinigten Staaten sehr hoch sind, erliegen die Verfassungsrichter zuweilen der Versuchung, die gut 200 Jahre alte Verfassung durch die ihnen übermittelte Kompetenz auf den »aktuellen « Stand zu bringen und der gesellschaftlichen Entwicklung anzupassen ${ }^{6}$. Die Kritik bezüglich solch richterlicher Anmaßung fällt in den Vereinigten Staaten besonders vehement aus ${ }^{7}$. Vor allem im Hinblick auf verfassungsrechtliche Fragen wie die der Legalisierung der Abtreibung, der Rechtmäßigkeit der affirmative action (Politik der Minderheitenförderung), des busings (die Beförderung von Schülern zu Bildungseinrichtungen in anderen Wohngebieten zum Ziel der » Rassenintegration«) sowie des Ausmaßes der Redefreiheit geht in der Bevölkerung und bei den Vertretern der politischen Parteien die Meinungen weit auseinander. Nicht selten werden besonders umstrittene Verfassungsfragen vom Supreme Court mit einer Mehrheit von 5-4 Stimmen entschieden. Unabhängig davon, wie das Urteil ausfällt, wird es immer eine »Spaltung « der amerikanischen Gesellschaft in verschiedene ideologische Lager nach sich ziehen. $\mathrm{Da}$ diese divergierenden Meinungen bezüglich eines höchstrichterlichen Urteils vom einfachen Bürger über den Politiker bis hin zum dissenting justice des Gerichts selbst reichen, verwundert es nicht, wenn bestimmte Verfassungsfragen immer wieder vor dem Supreme Court verhandelt werden. Dies gilt umso mehr, da insbesondere pressure groups daran interessiert sind, bestimmte Urteile zu revidieren $^{8}$. Die Flexibilität des anglo-amerikanischen Rechtes, das in erster Linie auf Präzedenzfällen beruht, ermöglicht diese Vorgehensweise?.

6 Die Meinungen scheiden sich daran, ob es die Aufgabe der Richter ist, gesellschaftliche Entwicklungen inner- und außerhalb der Vereinigten Staaten in die Rechtsprechung mit einfließen zu lassen. Die Supreme Court Kolumnistin der New York Times Linda Greenhouse hat dies am Ende der Sitzungsperiode 2002/03 folgendermaßen zum Ausdruck gebracht: "The Supreme Court that ended last week will leave as big an imprint as any in recent memory not only on the country, but on the court itself in ways few could have expected when the term began. The justices [...] they have displayed a new attentiveness to legal developments in the rest of the world and to the court's role in keeping the United States in step with them ". Linda Greenhouse, »In a Momentous Term, Justices Remake the Law, and the Court« in: The New York Times, 1. 7. 2003.

7 Viele, der bis zum heutigen Tage stark in die Tradition der Volkssouveränität verwurzelten US-Amerikaner lehnen den Eingriff, der nur indirekt legitimierten Richter, in die durch demokratische Mehrheiten verabschiedeten Gesetze strikt ab.

$8 \mathrm{Zu}$ den Interessengruppen, die gezielt auf den Supreme Court einwirken gehören unter anderem die ACLU (American Civil Liberties Union), die NAACP (National Association for the Advancement of Colored People) und Pro-Life America (Interessengruppe, die sich die Abschaffung der Abtreibung zum Ziel gesetzt hat). Da der Zug durch die Instanzen durchaus kostspielig ist, unterstützen die pressure groups Klagen, wenn diese ihrer jeweiligen Zielsetzung dienlich sind.

9 In den Vereinigten Staaten können sich die Richter auf diverse Rechtsquellen berufen. Dabei wird in erster Line zwischen dem geschriebenen Recht und dem Richterrecht unterschieden. $\mathrm{Zu}$ dem geschriebenen Recht gehört neben dem constitutional law, das statutory law, administrative law auch das common law. Vgl. Emil Hübner, Das politische System der USA, 5., aktualisierte Auflage, München 2003, S. 151. 
Im Rahmen dieser Arbeit werden im Wesentlichen folgende Fragen erörtert: Inwiefern gab es innerhalb der letzten 50 Jahre einen Wandel in der Rechtsprechung des Supreme Court? Wie lässt sich ein möglicher Wandel an verschiedenen Urteilen der einzelnen »Gerichte « vergleichend feststellen? Hat sich die konservative Forderung nach richterlicher Zurückhaltung durchsetzen können?

Dabei soll die Entwicklung in der Rechtssprechung des Obersten Bundesgerichts seit Mitte der 50er Jahre skizziert werden. Hierbei sollen explizit Verfassungsfragen im Gebiet der Gleichheit vor dem Gesetz, im Besonderen die rechtlichen Auswirkungen, die das Ende der »Rassentrennung « im Süden der Vereinigten Staaten mit sich brachte, behandelt werden. Die daraus abgeleitete Gesetzgebung von gleichheitsfördernden Maßnahmen, die unter dem Sammelbegriff affirmative action bekannt wurde, gewann letztlich über den Süden hinaus für das gesamte Land an Relevanz. Zudem wird die Interpretation des Persönlichkeitsrechts und der bei der Strafverfolgung relevanten Artikel der amerikanischen Bill of Rights durch die einzelnen »Gerichte« erörtert. Beginnend mit dem als sehr liberal geltenden Warren Court, über den als moderat eingestuften Burger Court, bis hin zum in vielen Bereichen als konservativ eingeordneten Rehnquist Court ${ }^{10}$ wird jedes dieser Gerichte im Einzelnen vorgestellt. Dies geschieht anhand einer Auswahl von Urteilen zu den oben angeführten Verfassungsfragen. Abschließend folgt eine Analyse der sich abzeichnenden Unterschiede in der Verfassungsrechtsprechung. Zudem soll ein kurzer Ausblick in die Zukunft des Obersten Gerichtshofes der Vereinigten Staaten gewagt werden.

Seit 1869 besteht das Gericht mit Ausnahmen kurzer Vakanz aus neun Richtern, deren Ansichten hinsichtlich der Verfassungsauslegung und politischen Überzeugungen divergieren, nicht selten konträr sind ${ }^{11}$, was folgendes Zitat über den Warren Court verdeutlicht: "Most of the issues coming to the Court allowed a merging of the views into a majority opinion, increasingly one by Brennan. But both because the justices were not interested in theory, and because their preferences varied, it is difficult to speak of an overriding jurisprudence apart from the results reached " ${ }^{12}$. Von einer Einheit des Supreme Court zu sprechen wäre demnach fern jeglicher Realität. Abstimmungen der Richter fallen bei Urteilen nur selten einstimmig aus. Die Divergenzen hinsichtlich der Verfassungsauslegung durch die einzelnen Supreme Court Richter lassen sich bei der Lektüre von jenen Urteilen deutlicher erkennen, die ne-

10 Wie oben bereits angedeutet, wird in dieser Arbeit natürlich keineswegs von einer Homogenität der richterlichen Meinungen während der einzelnen Gerichtsären ausgegangen. Die Benennung des Gerichtes nach dem jeweiligen Vorsitzenden soll dies auch keinesfalls suggerieren. Von der Behauptung, die Rechtssprechung dieser Ären, entspreche jeweils durchgängig den juristischen Ansichten ihrer Vorsitzenden, gilt es ebenfalls Abstand zu nehmen. G. Edward White, The American Judicial Tradition. Profiles of Leading American Judges, Oxford u. a. 1976, S. 317-325.

11 Selbst die Einteilung der Richter in das liberale, moderate oder konservative Lager ist eher grob und berücksichtigt nur selten die grundlegenden Meinungsverschiedenheiten innerhalb dieser Lager bei den verschiedenen Verfassungsfragen, die an das Gericht herangetragen werden.

12 Lucas A. Powe Jr., The Warren Court and American Politics. History's Warren Court: The 1962-1968 Terms, Cambridge u. a. 2000, S. 209-460, S. 216. 
ben der Mehrheitsauffassung ${ }^{13}$ (opinion of the court) stets concurring opinions beziehungsweise dissenting opinions der Richter beinhalten ${ }^{14}$.

\section{Warren Court 1953-1969}

Die Durchsetzung von fundamentalen Grundrechten, im Besonderen des Prinzips der Gleichheit - hier vor allem die Feststellung der Verfassungswidrigkeit der Segregation - kennzeichnete den Warren Court. Bereits zu Anfang der Warren-Ära fiel ein Urteil, das als das wichtigste des 20. Jahrhunderts eingestuft wird. Die Entscheidung Brown v. Board of Education of Topeka $(1954)^{15}$ war der Auftakt für eine progressive Phase amerikanischer Verfassungsgerichtsbarkeit, die bislang ihresgleichen sucht. Inwieweit der Warren Court mit seinen Entscheidungen liberale Politik betrieben oder nur die Grundsätze der amerikanischen Verfassung auf eine elementare und angemessene Art und Weise zeitgemäß interpretiert hat, ist bis heute heftig umstritten und wird wohl auch in der Zukunft Bestandteil von Diskussionen und wissenschaftlichen Arbeiten bleiben. Die historische Entscheidung Brown v. Board of Education of Topeka $I^{16}$ von 1954, in deren Urteilsbegründung der Supreme Court die »Rassentrennung « in öffentlichen Schulen für verfassungswidrig erklärte, betraf - über die fünf klagenden Parteien hinaus - die gesamte Gesellschaft in den Vereinigten Staaten, da sich die Aufhebung der »seperate but equal«-Doktrin bald auf alle Bereiche des öffentlichen Lebens ausgeweitet wurde ${ }^{17}$. Die Entscheidung beschränkte sich nicht nur auf die anhand von Gesetzen ${ }^{18}$ vormals »legal « segregierten Südstaaten, sondern sie zog, mit einer gewissen zeitlichen Verzögerung, ebenso

13 Die opinion of the court spiegelt die Entscheidung des behandelten Falles wider. Daneben haben die Richter, die mit der Mehrheit gestimmt haben, das Recht, eine conncurring opinion zu verfassen, wenn sie mit einzelnen Argumentationsteilen des Urteils nicht übereinstimmen. Richter, die bei einer Urteilsabstimmung in der Minderheit waren, haben anhand der dissenting opinion die Möglichkeit, ihre rechtliche Sichtweise des Falles zum Ausdruck zu bringen und die Argumente niederzulegen, die sie veranlasst haben, gegen das Mehrheitsurteil zu votieren. Henry J. Abraham, The Judiciary. The Supreme Court in the Governmental Process, 9. Auflage, Madison et al. 1993, S. 31 ff.

14 In der Regel werden 1/4 bis 1/3 der Urteile des Gerichts einstimmig getroffen. Ebd., S. 32.

15 Die Brown Entscheidung stärkte nicht nur die Bürgerrechtsbewegung der Afroamerikaner und die anderer Minderheitsgruppen. Sie markierte auch den Anfang des vielfach heftig kritisierten »liberalen Aktivismus « des Warren Courts. Glaubt man der unter vielen Experten geteilten Meinung, so setzte Brown den Maßstab für die nachfolgenden Urteile des Gerichts unter Warren.

16347 U. S. 483 (1954). Vgl. Winfried Brugger, Grundrechte und Verfassungsgerichtsbarkeit in den Vereinigten Staaten von Amerika, Tübingen 1987, S.144-162, S. $152 \mathrm{ff}$.

17 Das Urteil war ein wichtiger Anstoß der Civil Rights Revolution, die Anfang der 60er Jahre ihren Höhepunkt erreichte. Diese bedingte die Verabschiedung des Civil Rights Act (1964), einer umfassenden Anti-Diskriminierungsgesetzgebung.

18 Gemeint ist das in den Südstaaten nach Ende des Civil War etablierte "System « der Apartheidsgesetze und -regeln (»Jim Crow Laws«), sowie der »black codes«, die eingeführt wurden um die Partizipation von Afroamerikaner bei Wahlen auszuschließen. 
Konsequenzen für die de facto nach Hautfarbe getrennten Nordstaaten ${ }^{19}$ nach sich. Durch sein einstimmig gefälltes Urteil in dem Brown-Fall verwarf das Gericht den Präzedenzfall Plessy v. Ferguson $(1896)^{20}$, in welchem es die »seperate but equal $\ll-$ Doktrin verfassungsrechtlich legitimiert hatte. Jedoch provozierte die Brown-Entscheidung innerhalb der Bevölkerung solch heftigen Widerstand, dass sich das Gericht dazu gezwungen sah, 1955 in einer zweiten Urteilsbegründung Brown v. Board of Education of Topeka II (1955) ${ }^{21}$ die in der ersten Entscheidung offen gelassenen rechtlichen Folgen, die das Urteil nach sich zog, auszuformulieren. So ordnete das Gericht in Brown II an, die Segregation im Schulsystem »with all deliberate speed « aufzuheben. Die Überwachung und Anleitung dieser Exekutivorder unterstellte das Gericht den jeweils zuständigen erstinstanzlichen Bundesgerichten ${ }^{22}$. Der fortwährende öffentliche Widerstand gegen die Umsetzung von Brown II zog noch mehrere Klagen gegen die andauernde »Rassentrennung « in Schulen nach sich, so den Fall Cooper v. Aaron (1958) ${ }^{23}$.

Ein weiteres grundlegendes Verfassungsprinzip im Bereich der equal rights, welches das Gericht ausführlich behandelte, war die Wahlrechtsthematik. Während der Supreme Court die Umsetzung des Wahlrechts früher allein den Einzelstaaten überlassen hatte und nur bei groben Verstößen gegen den 15. und 19. Zusatzartikel der Verfassung einschritt, änderte sich dies unter dem Warren Court ${ }^{24}$. Als der Fall Reynolds v. Sims ${ }^{25}$ im Jahre 1964 vor dem Gericht verhandelt wurde, hatte sich das Ge-

19 Gemeint ist die in den Nordstaaten der Vereinigten Staaten vorherrschende institutionelle Segregation. Die Brown-Entscheidung hatte auf diese Art der Segregation keine sofortigen Auswirkungen, erst das Jahre später angeordnete, auf der Brown-Entscheidung beruhende busing, wirkte sich auf diese Problematik der Segregation aus (Keyes $v$. School District No. 1, Denver, 413 U.S. 189 (1973)). Alfred H. Kelly u.a., The American Constitution, Its Origins and Development Band II, 7. Auflage, New York/London 1991, S. 678-704, S. 684.

20 Brugger, Grundrechte und Verfassungsgerichtsbarkeit, aaO. (FN 16), S. 144 -152, S. 150.

21 Ebd., S. 154.

22 Auch die Anweisung Chief Justice Earl Warrens, die Desegregation »with all deliberate speed $\ll$ durchzuführen, resultierte vorwiegend in Stillstand oder ausgeprägten Widerstand. Ferner wurde die Entscheidung von vielen Seiten deswegen kritisiert, weil sie vor allem weiße Südstaatler geradezu zur Nichteinhaltung provozieren würde. Engstirniger Widerstand war die Norm, so dass zehn Jahre nach Brown I im Süden erst 1,2\% aller afroamerikanischen Kinder integrierte Schulen besuchten. Abraham L. Davis / Barbara Luck Graham, The Supreme Court, Race, and Civil Rights, Thousand Oaks u. a. 1995, S. 117-128, S. 125.

23 Im Urteil zu dem genannten Fall betonte das Gericht, dass der VI. Artikel der Verfassung diese zum "supreme law of the land " macht und folglich die Brown Entscheidung für alle Einzelstaaten bindend sei. Aber selbst die Berufung auf den als »national supremacy bekannten Verfassungsgrundsatz änderte am Widerstand der Desegregationsgegner nur wenig, was in der Konfrontation in Little Rock besonders deutlich zu Tage trat. Ebd., S. 127.

24 Das Gericht nahm bis zu diesem Zeitpunkt also keinerlei Anstoß an bestimmten einzelstaatlichen Regelungen, wie zum Beispiel den so genannten »black codes", die insbesondere im Süden eine Wahlsteuer, Lese- und Schreibtests, eine bestimmte Wohnsitzdauer oder ein bestimmtes Wahlalter vorschrieben. Brugger, Grundrechte und Verfassungsgerichtsbarkeit, aaO. (FN 16) S. 77-84, S. 77.

25 Reynolds v. Sims, 377 U. S. 533 (1964). Ebd. 
richt bereits in andere Streitigkeiten über die Neueinteilung von Wahlkreisen eingeschaltet, und somit die oben beschriebene Zurückhaltung seiner Vorgänger aufgegeben ${ }^{26}$. Die Urteilsbegründung des Gerichts im Fall Reynolds v. Sims »one man, one vote", die auf die Umsetzung des allgemeinen und gleichen Wahlrechts abzielte, ging über die in der Verfassung verankerten Einzelvorschriften des Wahlrechts hinaus ${ }^{27}$. Earl Warren, der das Wahlrecht als fundamentales Recht ansah, erachtete seine Argumentation indes durch den im 14. Zusatzartikel enthaltenen due process und die equal protection clause bestätigt: "Undoubtedly, the right of suffrage is a fundamental matter [377 U.S. 533, 562] in a free and democratic society. Especially since the right to exercise the franchise in a free and unimpaired manner is preservative of other basic civil and political rights, any alleged infringement of the right of citizens to vote must be carefully and meticulously scrutinized «28. Die "Apportionment Revolution «, lässt sich nach der Meinung Alfred H. Kelly folgendermaßen einordnen: "Apart from its leadership in the black revolution, the most significant egalitarian reformist activism in which the Warren Court engaged was its imposition of the 'one man, one vote' principle upon representation in state legislature and in Congress ${ }^{29}$. Der Warren Court setzte auch im Bereich der privacy rights neue Maßstäbe. Noch im Jahr 1967 gab es in 16 Einzelstaaten Gesetze, die »interracial marriages «, also Ehen zwischen Weißen und Afroamerikanern, unter Strafe stellten. In diesem Jahr wurde der Fall Loving v. Virginia vor dem Supreme Court entschieden $^{30}$. Chief Justice Warren brachte in seiner Urteilsbegründung eindringlich zum Ausdruck, dass kein Staat das Recht besitze, einer Person das individuelle Recht, einen Menschen von anderer Hautfarbe zu heiraten, abzuerkennen, da das Recht der

26 Bei der Verhandlung Baker v. Carr, 369 U.S. 186 (1962) erkannte der Supreme Court zum ersten Mal die Justiziabilität von Erfolgswertfragen nach der equal protectionKlausel an. Ebd., S. 80; In Gray v. Sanders, 372 U.S. 368 (1963) entschied das Gericht, dass der 14. Zusatzartikel den one-person-one-vote Standard impliziert. Mit der Entscheidung Wesberry v. Sanders (1964) wandte das Gericht diesen Standard zum ersten Mal auf bundesweite Wahlen an (House of Representatives). Damals basierte seine Begründung jedoch noch weitgehend auf Artikel I der Verfassung und noch nicht auf dem 14. Zusatzartikel. Diese Entscheidungen legten die »Grundsteine « für die Reynolds-Entscheidung und die Interpretation des Gerichts wonach der one-man-one-vote Standard durch die equal protection-Klausel des 14. Zusatzartikels garantiert sei. Sheldon Goldman, Constitutional Law: Cases and Essay, 2. Auflage, New York u. a. 1991, S. 788-790.

27 Kritiker der Entscheidung beanstandeten neben der aktiven Rolle der Urteilsformulierung des Gerichts auch die bereits bei Brown II angetroffene Anordnung der Überwachung der Urteilsumsetzung durch das Gericht selbst, beziehungsweise durch die ihm unterstehenden Bundesgerichte, da sie Genanntes als Usurpation sowohl legislativer wie auch exekutiver Kompetenz ansahen. Christopher Wolfe, The Rise Of Modern Judicial Review. From Constitutional Interpretation to Judge-Made Law, Lanham 1994, S. 266.

28 »Opinion of the Court«, Reynolds v. Sims, 377 U. S. 533 (1964).

29 Alfred H. Kelly, The American Constitution, aaO. (FN 19), S. 614.

30 Bereits im Jahr 1964 hob das Gericht ein Statut des Staates Florida auf, welches es »interracial couples" verbat zusammen zu wohnen, geschweige denn eine Nacht in ein und demselben Raum zu verbringen McLaughlin v. Florida, 379 U. S. 184 (1964). 
Heirat zu den fundamentalen Rechten eines jeden Individuums gehör $\mathrm{t}^{31}$. Deswegen unterliege dieses Recht dem Schutz der Privatsphäre und dürfe vom Staat nicht angetastet werden.

Auf dem Gebiet der Delinquentenrechte und der Todesstrafe schuf das Gericht unter dem Vorsitz von Earl Warren besonders im erstgenannten Bereich grundlegende Präzedenzfälle. Durch seine Auslegung der Zusatzartikel der Verfassung, welche die Reglementierung der Strafverfolgung festlegen (sprich den Rechtsschutz, der mutmaßlichen Tätern zusteht), kam es zu Kontroversen. Besonders umstritten war das Urteil im Fall Miranda v. Arizona (1966). Die Entscheidung, welche die Polizei zwingend auf die Nennung der Rechte bei jeder Verhaftung festlegt, stellt sowohl die bekannteste als auch am stärksten kritisierte Entscheidung des Warren Courts in Sachen Strafverfolgung dar ${ }^{32}$. Nach Meinung der Mehrheit des Gerichts bildet die Aufklärung über die Rechte ${ }^{33}$ das notwendige Gegengewicht zu der repressiven Atmosphäre in Polizeirevieren. Zwar bleibe es dem Verhafteten offen gestellt, diese Rechte in Anspruch zu nehmen, jedoch müsse die Verteidigung im Falle eines Verzichtes nachweisen, dass dieser freiwillig, wissentlich und mit Vernunft getroffen wurde. Die Entscheidung stieß auf harsche Kritik von Polizisten, Strafverfolgern und öffentlichen Vertretern aller Regierungsebenen ${ }^{34}$. Bereits 1961 hatte das Gericht mit seinem Urteil in Mapp v. Ohio für Aufsehen gesorgt, als es die 1914 von ihm selbst etablierte exclusionary rule ${ }^{35}$ vom Bundes- auf die Einzelstaaten ausweitete. Die Regel verbietet die Zulassung der von den Ermittlungsbehörden illegitim erlangten Beweise vor Gericht ${ }^{36}$. In der Entscheidung Gideon v. Wainwright (1963) weitete das Gericht das im 6. Zusatzartikel garantierte Recht auf anwaltlichen Beistand aus. Das Recht, das zum damaligen Zeitpunkt nur in Fällen von Kapitalver-

31 Vgl. Davis, The Supreme Court, aaO. (FN 22), S. 160-161.

32 Kenneth Jost, »Miranda v. Arizona in: Kenneth Jost, The Supreme Court A to Z, 2. Auflage, Chicago/London 1998, S. 283.

33 Die so genannten Miranda Warnings: "You have the right to remain silent. Anything you say can and will be used against you in a court of law. You have the right to have an attorney present before any questioning. If you cannot afford an attorney, one will be appointed to represent you before any questioning".

34 Der spätere Präsident Richard Nixon nutzte die Empörung für die Durchführung seines erfolgreichen Wahlkampfes, indem er "law and order" propagierte und versprach Richter zu ernennen, die mehr Sympathie für die Polizei aufweisen und weniger über die Rechte von Angeklagten besorgt seien. Jost, »Miranda v. Arizona« aaO. (FN 32), S. 283.

35 Dies wurde möglich durch die schon seit geraumer Zeit anzutreffende Interpretation des Gerichtes, dass die im Sinne des due process wesentlichen Bestimmungen der Bill of Rights als Verfassungsgarantien auch gegenüber Übergriffen der Einzelstaaten als bindend erachtet werden. Vgl. Horst Dippel, »Bill of Rights « in: Rüdiger B. Wersich (Hg.), USA-Lexikon. Schlüsselbegriffe zu Politik, Wirtschaft, Gesellschaft, Kultur, Geschichte und zu den deutsch-amerikanischen Beziehungen, Berlin 1996, S. 128-129.

36 Neben dem Verbot von Beweisen, die anhand eines Verstoßes gegen den 4. Zusatzartikel (search and seizure) erlangt wurden, verbot das Gericht zudem die Verwendung von Aussagen, die unter Verstoß gegen das im 5. Zusatzartikel genannte Recht gegen Selbstbeschuldigung (self-incrimination) oder das im 6. Artikel fixierte Anrecht auf Rechtsbeistand zustande kamen. Vgl. Claus D. Rade, »Due Process of Law « in: ebd., S. 237239. 
brechen seine Anwendung fand, galt von da an für alle Anklagen, die eine Gefängnisstrafe nach sich ziehen können ${ }^{37}$.

Nach den genannten Entscheidungen des Warren Court mag man sich fragen, warum die Thematik des capitel punishment in den Jahren der Warren Ära nie zur Disposition stand. Das einzige Urteil, das zu diesem Kontext fiel, beschränkte sich auf die Auswahl von Geschworenen in Todesstrafprozessen. In Witherspoon v. Illinois (1968) entschied das Gericht, dass auch Todesstrafengegner in solchen Prozessen als Geschworene zulässig seien.

\section{Burger Court 1969-1986}

Selbst Jahre nach der Verabschiedung des Civil Rights Act durch den Kongress beschäftigten Fragen der Gleichberechtigung und der Integration von Afroamerikanern den Supreme Court, zumal die Fortschritte im Feld der Eingliederung vormals »rassisch « getrennter Schulsysteme äußerst schleppend vorankamen. In der Entscheidung Swann v. Charlotte-Mecklenburg Board of Education (1971) urteilte der Burger Court, dass das busing als ein adäquates Mittel im Kampf gegen ein duales Schulsystem eingesetzt werden kann ${ }^{38}$. Zwei Jahre später entschied das Gericht seinen ersten Fall außerhalb des Südens, welcher die de facto Segregation in Denver thematisierte. Das Urteil in Keyes v. School District No. 1, Denver, Colorado (1973) besagte: "that purposeful discrimination is adequate for an inferential finding that de jure segregation is systemwide «39. In der Urteilsbegründung ging das Gericht »aufs Ganze « und ordnete zum ersten Mal das Verfahren des busings in einer Stadt im Norden an. Diese Entscheidung blieb aufgrund des weit verbreiteten Widerstands gegen das busing vorerst die Einzige, die den Norden der Vereinigten Staaten betraf. Trotz des omnipräsenten Widerstandes in der amerikanischen Bevölkerung gegen

37 Mit diesem Urteil hob das Gericht die Entscheidung Betts v. Brady, 316 U.S. 455 (1942) auf. Damals hatte das Gericht entschieden, dass das in der Bill of Rights garantierte Recht auf einen Verteidiger (6. Amendment) nicht zu den fundamentalen Rechten der Verfassung gehöre. Mit seinem Urteil bestätigte es aber wiederum einen Präzedenzfall, der weiter zurücklag als Betts v. Brady: "We think the Court in Betts was wrong, however, in concluding that the Sixth Amendment's guarantee of counsel is not one of these fundamental rights. Ten years before Betts v. Brady, this Court [. . .] had [. . .] declared that 'the right to the aid of counsel is of this fundamental character.' Powell $v$. Alabama, [...] (1932). While the Court at the close of its Powell opinion did [. . .] limit its holding to the particular facts and circumstances of that case, its conclusions about the fundamental nature of the right to counsel are unmistakable". Opinion of the Court, Gideon v. Wainwright, 372 U.S. 335 (1963).

38 Vgl. Charles M. Lamb/Stephen C. Halpern (Hg.), The Burger Court. Political and Judicial Profiles, Urbana u. a. 1991, S. 18-34, S. 21.

39 Folglich sah das Gericht den Tatbestand der de jure Segregation dann für das ganze Schulsystem als gegeben an, sobald er nur in einem Bereich des Schulsystems nachgewiesen wurde. Keyes v. School District No. 1, Denver, Colorado, 413 U.S. 189. 
das busing bestätigten die Urteile des Supreme Court mit einer Ausnahme ${ }^{40}$ bis 1976 die durch die Kommunen oder untergeordneten (Bundes-) Gerichte angeordneten busing-Pläne als Mittel im Kampf gegen »Rassentrennung " an Schulen ${ }^{41}$. Im Jahr 1976 begann dann, unter anderem bedingt durch weitere Neubesetzungen, die bisherige Front der Befürworter dieser Maßnahme am Obersten Gerichtshof zu bröckeln und als Folge dessen votierte das Gericht verstärkt gegen integrative Maßnahmen ${ }^{42}$. Gleichwohl fielen bis ins Jahr 1982 noch Entscheidungen für den »Transfer « von Schulkindern in andere Wohngebiete, wenn auch meist auf der knappest möglichen Entscheidungsgrundlage von 5-4 Stimmen.

Eine weitere Maßnahme, die zum Ausgleich der Segregation, Diskriminierung und Ungleichbehandlung von Afroamerikanern (sowie von anderen Minderheiten) etabliert wurde, war die Erhöhung des Anteils dieser Gruppen auf eine möglichst repräsentative Zahl in allen Arbeitsbereichen, einschließlich der Zulassung an Universitäten. Diese unter dem Sammelbegriff affirmative action bekannt gewordenen Programme zur Förderung ethnischer Minderheiten und Frauen waren im Burger Court seit jeher umstritten ${ }^{43}$. In Griggs v. Duke Power Co. (1971) bestätigte das Gericht noch Abschnitt VII des Bürgerrechtsgesetzes und machte somit affirmative action bindend. Dabei beschränkte sich das Gericht nicht allein darauf, Regelungen von Arbeitgebern, denen die Intention der Diskriminierung explizit nachgewiesen werden konnte, strafbar zu machen, sondern stellte auch Regelungen, die diskriminierende Nebenfolgen nach sich ziehen können, unter Strafe ${ }^{44}$.

40 In Miliken v. Bradley, 418 U.S. 717 (1974), lehnte das Gericht einen umfassenden Plan $\mathrm{ab}$, welcher das zwischen den innerstädtischen und vorstädtischen Distrikten herrschende Ungleichgewicht von afroamerikanischen und weißen Schülern ausgleichen sollte. Der Grund der Ablehnung lag darin, dass das Gericht keinen Beleg dafür ausmachen konnte, dass die vorstädtischen Bezirke zu der Segregation beigetragen hatten. Davis, The Supreme Court aaO. (FN 22), S. 224; Kelly, The American Constitution, aaO. (FN 19), S. 685.

411971 brachte das Gericht zudem noch ein Gesetz des Staates North Carolina zu Fall, welches das busing und die Zuweisung von Schülern an Schulen zugunsten der Desegregation verbot. North Carolina State Board of Education v. Swann, 402 U.S. 43 (1971). Davis, The Supreme Court, aaO. (FN 22), S. 219-226.

42 Fälle, in denen das Gericht gegen integrative Maßnahmen stimmte, waren: Pasadena City Board of Education v. Spangler, 427 U.S. 424 (1976), Metropolitan School District v. Buckley, 429 U.S. 1068 (1977) sowie Dayton Board of Education v. Brinkman, 433 U.S. 406 (1977). Ebd., S. 225.

43 Anhand der 1965 und 1968 durch den Kongress verabschiedeten Ausführungsverordnungen des Civil Rights Act von 1964 wurde allen Unternehmen, die mit dem Staat zusammenarbeiten, die Auflage gemacht, Angehörige ethnischer Minderheiten und Frauen bevorzugt einzustellen und zu befördern. Dem folgten weitere Verordnungen, unter anderem auch für den öffentlichen Dienst und Ausbildungsinstitutionen. Helgard Kramer, »Affirmative Action« in: Wersich, USA-Lexikon, aaO. (FN 35), S. 20-23.

44 Das Gericht erkannte die statistische Beweisführung in affirmative action-Fällen als legitim an, mit deren Hilfe die (am weitesten verbreitete) indirekte Diskriminierung bewiesen werden kann. Vgl. ebenda, S. 21. 
So dauerte es nicht lange, bis Klagen von weißen Männern an das Gericht herangetragen wurden, die sich aufgrund der Politik der Minoritätenförderung der »umgekehrten Diskriminierung « ausgesetzt sahen. Eines der wichtigsten Urteile in dieser Hinsicht wurde im Fall Regents of University of California v. Bakke (1978) getroffen. Im genannten Rechtsstreit hatte ein Student geklagt, welcher zweimal am Zulassungsverfahren der kalifornischen Universität gescheitert war. Er wandte sich gegen die Regelung der Universität, 16\% der Studienplätze anhand einer affirmative action-Maßnahme zu vergeben, selbst wenn die Bevorzugten geringere Leistungen bei den Eingangstests erreicht hatten als ihre weißen Mitbewerber ${ }^{45}$. Der Supreme Court sprach dem Kläger ein Recht auf Zulassung zu und erklärte Teile des Sonderzulassungsverfahren der Universität für verfassungswidrig ${ }^{46}$. In Fullilove v. Klutznick (1980) hielt der Supreme Court den Public Works Employment Act (1977) des Kongresses aufrecht, nach welchem die Festlegung der Vergabe von mindestens $10 \%$ der bundesstaatlichen Mittel bei öffentlichen Bauvorhaben an Firmen gehen müssen, die im Besitz von Angehörigen einer »rassischen « oder ethnischen Minderheit sind. Das Gericht wies die Klage weißer Bauunternehmer mit der Begründung $\mathrm{ab}$, dass der Kongress in Ausübung seiner in Artikel I, Absatz 8 genannten Autorität gehandelt habe ${ }^{47}$. Ab 1984 fielen die Entscheidungen des Gerichts in Fragen der Minderheitenförderung in der Arbeitswelt deutlich restriktiver aus. In dem Fall Firefighters Local Union No. 1784 v. Stotts (1984) entschied das Gericht gegen eine affirmative action-Maßnahme und gab der seniority rule Vorrang ${ }^{48}$. Die oben angeführten Urteile machen deutlich, dass das Gericht über die affirmative action-Frage tief gespalten war ${ }^{49}$. Zudem wuchs der Druck seitens der Exekutive, die Förderung von Minderheiten zu einem Relikt der Vergangenheit zu machen ${ }^{50}$.

45 Bakke sah damit eine Verletzung seiner Rechte unter der equal protection clause des 14. Zusatzartikels, sowie Artikel VI, Sect. 601 des Civil Rights Acts von 1964 gegeben. Brugger, Grundrechte und Verfassungsgerichtsbarkeit, aaO. (FN 16), S. 183.

46 Jedoch gelang es dem Gericht nicht, sich auf eine Mehrheitsmeinung in diesem Fall zu einigen. Strittige Punkte, für die sich keine Mehrheit fand, waren die Festlegung der verfassungsrechtlichen Anforderungen der equal protection clause speziell bei Sonderzulassungsverfahren, sowie auf einen entsprechenden Prüfungsmaßstab des Gerichtes bezüglich solcher Fälle. Vgl. ebd.

47 Zudem wies Chief Justice Burger in seiner Urteilsbegründung darauf hin: »Congress could have achieved its objectives by use of its power under $\int 5$ of the Fourteenth Amendment 'to enforce, by appropriate legislation' the equal protection guarantee of that Amendment«. Opinion of the Court, Fullilove v. Klutznick, 448 U.S. 448 (1980).

48 Kenneth Jost, "Affirmative Action « in: Jost, The Supreme Court A to Z, aaO. (FN 32); Kelly, The American Constitution, aaO. (FN 19), S. 729-753, S. 744.

49 Das Problem dabei war, dass gerade durch diese Spaltung nie ein Prüfungsmaßstab entwickelt wurde, der hätte festlegen können, welchen Kriterien affirmative action-Programme entsprechen müssen, um als legitim zu gelten.

50 Die Reagan Administration unterstützte mit Beginn ihrer Amtszeit Klagen von Weißen gegen die reverse discrimination (die sogenannte umgekehrte Diskriminierung) durch amicus curiae. Zudem hatte sie durch ihre Besetzungspolitik der Bundesgerichte klar Stellung gegen affirmative action genommen. Helgard Kramer, »Affirmative Action « in: Wersich, USA-Lexikon, aaO. (FN 35), S. 20-23. 
Seine wohl überraschendsten und liberalsten, aber auch umstrittensten Entscheidungen auf dem Feld der Persönlichkeitsrechte fällte das Gericht in der Problematik der Abtreibung. Die Tendenz in den Fällen Roe v. Wade (1973) und Doe v. Bolton (1973) manifestierte eine moderate Reformtendenz des Gerichts in dieser brisanten gesellschaftlichen Streitfrage. Durch die beiden Urteile des Supreme Courts wurden Gesetze zweier Einzelstaaten für nichtig erklärt, die Abtreibung unter Strafe stellten. Das Gericht entschied, dass die Gesetze gegen den 14. Zusatzartikel verstießen, da sie einen unrechtmäßigen Eingriff in die Privatsphäre von Frauen darstellten. Während das Gericht die oben genannten Urteile bis zum Ende der Amtszeit Earl W. Burgers unangetastet ließ, verringerte es doch die Möglichkeiten mittelloser Frauen auf Abtreibung. Dadurch, dass es sich für die Aufrechterhaltung ${ }^{51}$ zweier Gesetze, je ein einzelstaatliches und ein bundesstaatliches, entschied, die das Verbot beziehungsweise die Einschränkung öffentlicher Geldmittel zur Finanzierung von Abtreibungen für Frauen des Medicaid-Programms vorschrieben, schränkte es deren Möglichkeit stark ein, eine Abtreibung vornehmen zu können.

Wie oben bereits angemerkt, hatte der Burger Court in weiten Bereichen das Recht auf Privatsphäre ähnlich ausgelegt wie der Warren Court, wenn auch sichtlich moderater. Die Änderung des moderaten Kurses hinsichtlich der privacy rights wurde 1986 sichtbar, als vor Gericht ein Einzelstaatengesetz, das Homosexualität unter Strafe stellte, auf seine Konstitutionalität zu prüfen war. In dem Urteil zu Bowers v. Hardwick (1986) entschied sich das Gericht für die Verfassungsmäßigkeit des Gesetzes und begründete dies damit, dass homosexuelle Aktivität zwischen zwei erwachsenen Männern nicht unter die verfassungsmäßige Garantie des Schutzes der Privatsphäre des 14. Zusatzartikels falle. Bei diesem Urteil, eines der letzten unter der Leitung von Chief Justice Burger Court, wird offensichtlich, dass das Gericht in seiner Rolle als Protektor von Minderheiten gegen staatliche Diskriminierung deutlich konservativere Maßstäbe anlegte. Der folgende Auszug aus dem Urteil verdeutlicht dies: "Sodomy laws should not be invalidated on the asserted basis that majority belief that sodomy is immoral is an inadequate rationale to support the lawes « ${ }^{52}$.

In Sachen Delinquentenrechte und Todesstrafe traf das Gericht anfangs liberale Entscheidungen, was sich im Laufe der Jahre ändern sollte. Die Schlüsseldoktrinen, die der Warren Court im Bereich der Strafverfolgung etabliert hatte, wurden vom Burger Court deutlich moderater angewendet. In Harris v. New York (1971) erklärte das Gericht belastende Aussagen, die unter Verletzung der Entscheidung Miranda v. Arizona erlangt wurden, im Gerichtsverfahren für zulässig. Dies gilt seither, wenn sie zur Klärung der Glaubwürdigkeit eines Angeklagten beitragen, der im Zeugenstand seine vormals getroffenen Aussagen widerruft ${ }^{53}$. Das Anrecht auf einen Rechtsbeistand blieb aber auch unter dem Burger Court bestehen ${ }^{54}$. Zudem stellte

51 Maher v. Roe, 432 U.S. 464 (1977) und Harris v. McRae, 448 U.S. 297 (1980). Kelly, The American Constitution, aaO. (FN 19), S. 696.

52 Opinion of the Court, Bowers v. Hardwick, 478 U.S. 186 (1986).

53 Vgl. Lamb / Halpern, The Burger Court, aaO. (FN 38), S.21.

54 Argersinger v. Hamlin, 407 U.S. 25 (1972). Ebd., S. 22. 
das Gericht 1980 klar, dass Polizisten auch dann einen Haftbefehl benötigen, wenn sie im Zuge einer routinemäßigen Festnahme eines Verbrechers sein Haus betreten ${ }^{55}$.

Neue Maßstäbe setzte das Gericht in erster Linie in der Streitfrage über die Verfassungsmäßigkeit der Todesstrafe. Die 1972 geweckte Hoffnung der Todesstrafengegner, dass auch in den Vereinigten Staaten die Todesstrafe bald ein Relikt grauer Vorzeiten werden würde, wurde vom Burger Court 1976 wieder zunichte gemacht. Zustande gekommen war diese Hoffnung mit dem Urteil Furman v. Georgia (1972), welches Einzelstaatengesetze für verfassungswidrig erklärte, da sie gegen den 8. Zusatzartikel der Verfassung verstoßen, der grausame und ungewöhnliche Strafen verbietet. Jedoch stellte das Gericht in diesem Urteil nicht die Verfassungswidrigkeit der Todesstrafe an sich fest, sondern erklärte vielmehr die momentane Auferlegung und Ausführung der Todesstrafe für nicht konstitutionell ${ }^{56}$. Doch bereits 1976 wurde das oben genannte Urteil durch die Entscheidung Gregg v. Georgia (1976) wieder aufgehoben. Diesmal entschied das Gericht für die Verfassungsmäßigkeit der Todesstrafestatuten des Staates Georgia und machte seine Entscheidungsgründe folgendermaßen fest: »The punishment of death for the crime of murder does not, under all circumstances, violate the Eighth and Fourteenth Amendments [...] The Eighth Amendment, which has been interpreted in a flexible and dynamic manner to accord with evolving standards of decency, forbids the use of punishment that is 'excessive' either because it involves the unnecessary and wanton infliction of pain or because it is grossly disproportionate to the severity of the crime [...] The existence of capital punishment was accepted by the Framers of the Constitution, and, for nearly two centuries, this Court has recognized that capital punishment for the crime of murder is not invalid per se « ${ }^{57}$. Obwohl der Burger Court die Verfassungsmäßigkeit der Todesstrafe nach der Entscheidung aus dem Jahre 1972 nie wieder in Frage gestellt hat, so war er doch maßgeblich daran beteiligt, ihre »Angemessenheit«im Rahmen des begangenen Verbrechens sowie ihre humane Anwendung zu gewährleisten. In weiteren Urteilen erklärte er zum Beispiel, dass die Todesstrafe eine unangemessene Strafe für Vergewaltigungsdelikte ${ }^{58}$ sei und verbot es, Geisteskranke ${ }^{59}$ hinzurichten.

55 Payton v. New York, 445 U.S. 573 (1980). Ebd., S. 23.

56 Sprich, das Gericht nahm bei diesem Urteil nicht zu der Todesstrafe an sich Stellung, sondern betonte vielmehr die rechtsstaatlichen Vorraussetzungen, die gegeben sein müssen, um eine faire und nichtdiskriminierende Auswahl der zum Tode Verurteilten zu genügen. Vgl. Brugger, Grundrechte und Verfassungsgerichtsbarkeit, aaO. (FN 16), S. 307.

57 Opinion of the Court, Gregg v. Georgia, 428 U.S. 153 (1976).

58 Coker v. Georgia, 433 U.S. 584 (1977). Vgl. Davis, The Supreme Court, aaO. (FN 22), S. $257-262$, S. 258.

59 Ford v. Wainwright, 477 U.S. 399 (1986). Ebd., S. 259. 


\section{Rehnquist Court seit 1986}

Auch mehr als dreißig Jahre nach dem Brown-Urteil beschäftigten Defizite im Bereich der Gleichberechtigung das Oberste Gericht. Spätestens Anfang der 80er Jahre wurde deutlich, dass ein wirklich desegregiertes Schulsystem wohl ein Ideal bleiben würde, zumal sich die Tendenz des späten Burger Courts, verstärkt gegen integrative Maßnahmen zu stimmen, auch im Rehnquist Court fortsetzte. Erst 1990 nahm das Gericht wieder einen Fall an, der sich mit dem Thema der school desegregation auseinander setzte. In Missouri v. Jenkins (1990) hob das Gericht die Verordnung eines Bezirksrichters auf, welche eine Steuererhöhung angeordnet hatte, um die Finanzierung eines Magnet School Desegregation Plan sicherzustellen. Ein Jahr später entschied der Rehnquist Court, dass einstmals segregierte Schuldistrikte von einer Integrationsverordnung auszunehmen seien, sobald sie erste Schritte unternommen hätten, um die negativen Auswirkungen früherer Diskriminierung zu beseitigen ${ }^{60}$. Mit dieser Entscheidung sprach sich der Court eindeutig gegen weitere busingMaßnahmen in dem betroffenen Bezirk aus. Im darauf folgenden Jahr bestätigte das Gericht die vorhergegangene Entscheidung in Freeman v. Pitts (1992) ${ }^{61}$. Für viele überraschend war die 1992 zustande gekommene Entscheidung in United Staates v. Fordice (1992). Mit deutlicher Mehrheit von 8-1 Stimmen kam das Gericht zu der Entscheidung, dass die Übernahme und die Ausführung »rassenneutraler « Zulassungs- und Einstellungsverfahren bei der Verwaltung von Colleges und Universitäten nicht unbedingt eine Erfüllung staatlicher affirmative action -Verpflichtungen darstellte, die notwendig waren, um ein vormals de jure segregiertes System zu integrieren ${ }^{62}$.

Auch in Sachen der Minderheitenförderung wurde die striktere Handhabung verfolgt, die sich bereits gegen Ende der Burger-Ära durchgesetzt hatte. Die ersten Entscheidungen des Rebnquist Court zur affirmative action fielen dennoch zugunsten der minderheitsfördernden Programme aus. Zudem ließ das Gericht in diesem Zusammenhang zum ersten Mal auch Klagen eines US-Bürgers arabischer Herkunft und von US-Bürgern jüdischen Glaubens $z^{63}$. Ab 1989 stieg jedoch die Zahl der Entscheidungen stetig an, in denen gegen affirmative action-Regelungen entschieden wurde ${ }^{64}$. Ferner erfolgte in diesem Jahr eine Zäsur bei affirmative action-Klagen. In dem Urteil Wards Cove Packing Co., Inc. v. Atonio (1989) verschob das Gericht die zu erbringende Beweislast bei Klagen bezüglich des Artikel VII des

60 Board of Education of Oklahoma City Public Schools v. Dowell, 498 U.S. 237 (1991). Ebd., S. 359-362, S. 360.

61 Das Gericht entschied in dem Fall, dass ein Bezirksgericht seine Überwachungsautorität auch dann aufgeben kann, wenn noch nicht alle Stufen des Schulintegrationsplanes erfüllt worden sind. Ebd., S. 359.

62 Das Gericht forderte den Staat Missouri in seinem Urteil dazu auf, Maßnahmen einzuleiten, um das duale Bildungssystem abzubauen. Vgl. Wolfe, The Rise Of Modern Judicial Review, aaO. (FN 27), S. 362.

63 Vgl. Davis, The Supreme Court, aaO. (FN 22), S. 370.

64 Martin v. Wilks, 490 U.S. 755 (1989). Ebd. 
Bürgerrechtsgesetzes vom Arbeitgeber auf den Arbeitnehmer ${ }^{65}$. Dem folgten noch mehrere Entscheidungen, in denen Diskriminierungsklagen abwiesen wurden. Dessen ungeachtet blieben die vom Burger Court geschaffenen Präzedenzfälle, welche die Politik der Minderheitenförderung an sich als verfassungsmäßig einstufen, erhalten. Auch wenn die Auflagen für die Programme deutlich strikter formuliert wurden, gab es daher auch weiterhin Urteile zugunsten von affirmative action ${ }^{66}$. Dazu zählt auch eines der beiden aktuellsten Urteile ${ }^{67}$, die kurz vor dem Ende der Sitzungsperiode 2002/03 des Supreme Court gefällt wurden. Die genannten Fälle gehen wie bereits der Bakke-Fall auf Klagen weißer Studentinnen zurück, welche die Universität von Michigan wegen der so genannten »umgekehrten Diskriminierung « verklagten, da die genannte Universität in ihren Auswahlverfahren Studienbewerber, die einer Minorität angehören, vorrangig berücksichtigte. Im ersten Fall wurde mit knapper Mehrheit für die Universität entschieden, da fünf der neun Richter der Meinung waren, dass die Zugehörigkeit zu einer Minderheit als ein Faktor von vielen bei Zulassungsverfahren an Universitäten zulässig sei ${ }^{68}$. Im zweiten Fall wurde mit 6-3 Stimmen zugunsten der Klägerin entschieden. An Anlehnung an den oben behandelten Präzedenzfall Regents of University of California v. Bakke aus dem Jahre 1978 sprach sich das Gericht gegen das Punktsystem der Universität von Michigan aus, welches an Angehörige von Minderheiten bei der Zulassung 20 Punkte zusätzlich vergab ${ }^{69}$. Da die Richter in ihrer Urteilsbegründung im Fall Grut-

65 Die von dem Gericht auferlegte Beweislasterbringung der Kläger wurde durch den 1991 vom Kongress verabschiedeten Civil Rights Act wieder aufgehoben.

66 Wenn auch die Mehrzahl der Fälle unter Chief Justice William H. Rehnquist gegen affirmative action und Diskriminierungsklagen entschieden wurden. Davis, The Supreme Court, aaO. (FN 22), S. 371.

67 Grutter v. Bollinger, 02-241 (2003) und Gratz v. Bollinger, 02-516 (2003) wurden am 23.06.2003 entschieden.

68 Laut der Richtermehrheit ist die Zielsetzung der Förderprogramme, die in den 70er Jahren entstanden sind, um historisches Unrecht (vor allem an Afroamerikanern) und die lange soziale Ungerechtigkeit teilweise auszugleichen, für die Vereinigten Staaten auch weiterhin notwendig. Grund dafür sind die fortwährende Präsenz von sozialer Ungerechtigkeit und ungleicher Startchancen, die dem Ziel der »equal opportunities" immer noch im Wege stehen. Vielerorts wurde die Entscheidung überraschend aufgenommen. Zwar fehlte es der Universität nicht an Unterstützern (neben dem Militär unterstützten auch Firmen wie Microsoft die Position durch amicus curiae Schreiben an das Gericht), so schien durch die eindeutige Parteinahme der Bush-Administration (ausgenommen der Außenminister Colin Powell) gegen die Beibehaltung der affirmative action der Vorteil bei den weißen Klägerinnen zu liegen. Vgl. Steven Greenhouse / Jonathan D. Glater, »Companies See Court Ruling as Support for Diversity « in: The New York Times, 24.06.2003.

$69 \mathrm{Um}$ als undergraduate student an der Universität zugelassen zu werden muss man eine Punktzahl von 150 erreichen. Die Richtermehrheit sah in der Vergabe von 20 ExtraPunkten aufgrund der »Rassen-«bzw. Minderheitszugehörigkeit, den Faktor der ethnischen Herkunft zu stark gewichtet. Im Laufe der Entscheidung wurden vielfach wenig Worte darüber verloren, dass Extra-Punkte nicht nur an Minderheitsangehörige verteilt werden, sondern auch an Bewerber mit außergewöhnlichen sportlichen Leistungen (20 Punkte) sowie an Bewerber die aus dem Staat Michigan stammen (10 Punkte). Dietmar Ostermann, »Das Oberste Gericht der USA nimmt die Politik in Pflicht, Minderheitenförderung überflüssig zu machen« in: Frankfurter Rundschau, 25.06.2003. 
ter v. Bollinger kein Reglement hinzufügten, das bestimmt, in welchen Situationen eine Politik zur Förderung von Minderheiten angebracht ist und wie weit die Bevorzugung von Minderheiten gehen darf, kann davon ausgegangen werden, dass diese Thematik bald wieder vor dem Gericht verhandelt werden wird.

Ebenso wie sein Vorgänger hatte das Gericht unter dem Vorsitz von William H. Rehnquist über die Legalität der Abtreibung zu entscheiden. Bis Ende der 80er Jahre hatte das Gericht konsequent legislative Versuche, die darauf abzielten, das Recht auf Abtreibung einzuschränken, revidiert ${ }^{70} .1989$ entschied das Gericht jedoch zugunsten eines restriktiven Gesetzes des Staates Missouri. Chief Justice Rehnquist argumentierte in dem Urteil zu Webster v. Redroproductive Health Services (1989), dass ein Staat das Recht besitze, ungeborenes Leben zu schützen. Nach Meinung der Richter schränke das Gesetz eine Abtreibung nicht übermäßig ein ${ }^{71}$.

Beim Thema Homosexualität weist der Rehnquist Court eine weniger restriktive Tendenz als sein Vorgänger auf, auch wenn seine Rechtssprechung nie darauf ausgelegt war, Homosexuellen den gleichen Schutz zukommen zu lassen, wie ihn andere vormals diskriminierte Minderheiten in den letzten Jahrzehnten erfahren hatten. In Romer v. Evans (1996) erklärte das Gericht einen Verfassungszusatz des Staates Colorado für verfassungswidrig. Der Zusatzartikel verbot es einzelstaatlichen und kommunalen Exekutivorganen Gesetze und Verordnungen zu erlassen, die Homosexuelle unter speziellen Schutz stellen. Folgendes Zitat von Kenneth Jost zeigt, wie das Urteil seiner Meinung nach zu bewerten ist: "Although gay rights advocates were heartened, the decision could not be read as a broad declaration of gay rights. Instead, it held only that the government could not single out homosexuals for less than equal treatment ${ }^{72}$. Trotz weiterer Versuche von gay rights Gruppen lehnte das Gericht es bis zum Beginn der Sitzungsperiode im Oktober $2002 \mathrm{ab}$, sich erneut dieser Thematik anzunehmen. Mit der Anhörung des Falles Lawrence v. Texas, 02102 (2003) nahm der Supreme Court dann erneut einen Fall zur Verhandlung an, in dem er die Verfassungsmäßigkeit eines Einzelstaatengesetzes zu prüfen hatte, welches homosexuellen Kontakt unter Strafe stellt. Anders als noch im Präzedenzfall Bowers v. Hardwick aus dem Jahre 1986 entschied das Gericht dieses Mal zu Gunsten der Kläger. In der Urteilsbegründung, die im Besonderen auf die Grundrechte von Homosexuellen als freie und gleiche Bürger einging, berief sich das Gericht in seiner 5-4 Entscheidung, den Bowers Präzedenzfall für obsolet zu erklären, auch auf

70 Mit Ausnahme der in Kapitel 3 im Bereich der privacy rights genannten Gesetze, die das Medicaid-Programm betrafen.

71 Das Gesetz schreibt vor, dass Ärzte, die eine Abtreibung ab der 20. Schwangerschaftswoche vornehmen, erst Tests durchführen müssen, die prüfen, ob der Fötus bereits lebensfähig ist. Zudem verbietet das Gesetz jegliche Verwendung öffentlicher Geldmittel und öffentlicher Einrichtungen zur Durchführung einer Abtreibung, es sei denn das Leben der Mutter ist in Gefahr. Kelly, The American Constitution, aaO. (FN 19), S. 740.

72 Kenneth Jost, »Gay Rights « in: ders., The Supreme Court A to Z, aaO. (FN 32), S.189-191. 
ein Urteil des Europäischen Gerichtshofs für Menschenrechte ${ }^{73}$. Anhand dieses Urteils wollte die Mehrheit belegen, dass die Entscheidung des Gerichts aus dem Jahre 1986 dazu geführt hat, dass die Vereinigten Staaten weit hinter der Rechtsentwicklung anderer westlichen Ländern zurücklagen und dies bei der Aufrechterhaltung des Präzedenzfalles auch weiterhin getan hätten. Insgesamt stimmten in diesem Fall schließlich sechs der neun Richter für die Kläger ${ }^{74}$.

Bei der Strafverfolgung setzte sich der Trend in Richtung einer engeren Verfassungsauslegung fort. Wie bereits der Burger Court hielt auch der Rehnquist Court an den Präzedenzfällen bezüglich der Rechte von Delinquenten fest. Zwar wandte er eine deutlich rigidere Auslegung der Miranda-Regelung an und weigerte sich, die Logik der Warren Court-Entscheidungen auf aktuelle Entwicklungen bei der Strafverfolgung auszudehnen ${ }^{75}$. So entschied das Gericht, dass auch Geständnisse von Geisteskranken zulässig seien und hielt in einem ähnlichen Fall die präventive Arrestregelung des Bail Reform Act von 1984 aufrecht $^{76}$. Auch die Auslegung der exclusionary rule des 4. Zusatzartikels fällt unter dem Rehnquist Court weniger rigide aus ${ }^{77}$. Zudem ist das Gericht darauf bedacht, den Einzelstaaten wieder mehr Macht zukommen zu lassen. In Sachen der verhältnismäßigen Bestrafung von Straftätern fiel im März 2003 ein Urteil, welches das "three strikes lawe des Staates Kalifornien aufrechterhielt ${ }^{78}$.

In der Todesstrafenthematik behielt der Rehnquist Court die 1976 eingeschlagene Linie bei. Die deutliche Mehrheit der Verfassungsrichter sieht die Todesstrafe durch die Verfassung legitimiert und fühlt sich zudem durch große Teile der Bevölkerung

73 Was vor allem von den Richtern die gegen das Urteil stimmten heftig kritisiert wurde. Sie beanstandeten, dass bei der Überprüfung der Verfassungsmäßigkeit eines Gesetzes allein die amerikanische Verfassung zu Rate gezogen werden dürfe. Darauf angesprochen verteidigte Justice Breyer die Entscheidung folgendermaßen: »Through commerce, through globalization, through the spread of democratic institutions, through immigration to America, it's becoming more and more one world of many different kinds of people' fubr er fort. 'And how they're going to live together across the world will be the challenge, and whether our Constitution and how it fits into the governing documents of other nations, I think will be a challenge for the next generations" zitiert nach: John H. Cushman Jr., "O'Connor Indicates She Will Remain on Court« in: The New York Times, 07. 07. 2003.

74 Die Richterin O'Connor stimmte in dem Urteil mit der Mehrheit in Lawrence v. Texas. In der Abstimmung die zur Aufhebung des oben angeführten Präzedenzfalles Bowers v. Hardwick durchgeführt wurde stimmte sie jedoch mit der Minderheit. Dies mag mitunter daran liegen, dass sie im Jahre 1986 in diesem Urteil für die Aufrechterhaltung des Sodomieverbots gestimmt hatte. Vgl. Linda Greenhouse, »Justices, 6-3, Legalize Gay Sexual Conduct in Sweeping Reversal in: The New York Times, 27.06.2003.

75 Im Fall Moran v. Burbine, 475 U.S. 412 (1986) ließ das Gericht ein Geständnis zu, das ohne die Verlesung der Miranda Warnings zustande gekommen war.

76 Colorado v. Connelly, 479 U.S. 157 (1986); United States v. Salerno, 481 U.S. 739 (1987). Kelly, The American Constitution, aaO. (FN 19), S. 730.

77 Anderson v. Creighton, 483 U.S. 635 (1987). Ebd., S. 731.

78 Ewing v. California, 000 U.S. 01-6978 (2003). Das Gesetz sieht lange Strafen vor, wenn ein Angeklagter zum dritten Mal verurteilt wird. So ist unter diesem Statut zum Beispiel ein bereits vorbestrafter Mann, welcher zweimal beim Diebstahl von Kinderfilmen erwischt wurde, zu einer Gefängnisstrafe von 50 Jahren verurteilt worden. Linda Greenhouse, »Week in Review Desk, March 2-8: National: 'Three Strikes' Law Upheld « in: The New York Times, 09. 03. 2003. 
in ihrer Meinung bestätigt. Verbot das Gericht unter Burger die Anwendung der Todesstrafe bei Geisteskranken, so ließ der Court unter Rehnquist Exekutionen von geistig Zurückgebliebenen $\mathrm{zu}^{79}$ - zumindest bis zu der Grundsatzentscheidung von 20028. Weiterhin erfuhren 1987 vor allem afroamerikanische Angeklagte einen Rückschlag, als das Gericht sich der Baldus-Studie verweigerte ${ }^{81}$. Zusätzlich schränkte der Rebnquist Court die Möglichkeiten von Habeas Corpus-Petitionen für Inhaftierte ein ${ }^{82}$ und ließ "victim impact statements" in Todesstrafprozessen $\mathrm{zu}^{83} . \mathrm{Zu} \mathrm{Be}-$ ginn dieses Jahres erklärte das Gericht die Hinrichtung von Verurteilten, die zur Tatzeit minderjährig waren, für verfassungswidrig. Bis zu diesem Zeitpunkt war es vielen Einzelstaaten möglich Delinquenten zum Tode zu verurteilen, die zum Zeitpunkt ihres Verbrechens minderjährig waren. In 22 der Staaten war die Altersgrenze hierfür das 16. Lebensjahr, in drei weiteren Staaten das 17. Lebensjahr ${ }^{84}$.

Im Juli 2004 wies das Gericht die Exekutive zum ersten Mal seit dem Erlass der Notstandsgesetzgebung in ihre Schranken. Es sprach Klägern (die in die nach den Anschlägen des 11. September im Rahmen der Anti-Terror-Richtlinien entstandenen Kategorie der »enemy combatants" fielen), die seit Ende des Jahres 2001 in Guantánamo Bay, Kuba festgehalten werden, das Recht auf richterliche Anhörung zu $\mathbf{u u}^{85}$. Dieses Recht wurde ihnen bisher von der Bush Regierung mit der Begründung vorenthalten, dass die Gefangenenlager auf Guantánamo nicht der amerikanischen Jurisdiktion unterliegen. Das Gericht widersprach dieser Interpretation der Administration $^{86}$. Des Weiteren verwies der Supreme Court den Präsidenten zwecks einer konkreten Ermächtigung, wie mit den Inhaftierten zu verfahren sei, an den Kongress. Diese klassische Ausbalancierung innerhalb des Systems der "checks and balances" ist von maßgeblicher Bedeutung für dessen Funktionieren. Festzuhalten bleibt, dass die Befugnis des Präsidenten, in Kriegszeiten »feindliche Kämpfer« (auch amerikanische Staatsbürger können unter diese Kategorie fallen) zu inhaftieren, von dem Gericht als verfassungsgemäß eingestuft wird ${ }^{87}$. Einzig und allein der Zeitfaktor, nach welchem die Inhaftierung ohne Zugang zu einem Gericht und rechtlichem Beistand bis zum Lebensende eines »feindlichen Kombattanten« andauern könnte, sorgte bei einigen Richtern für Unbehagen ${ }^{88}$.

79 Penry v. Lynaugh, 492 U.S 302 (1989).

80 Linda Greenhouse, »The Supreme Court: The Death Penalty; Citing 'National Consensus', Justices Bar Death Penalty For Retarded Defendants « in: The New York Times, 21. 06. 2002.

81 McCleskey v. Kemp (1987). Die Studie zeigte auf, dass die Wahrscheinlichkeit, dass ein Afroamerikaner für den Mord an einem Weißen zum Tode verurteilt wird, um ein Vielfaches höher ist als die für einen Weißen. Davis, The Supreme Court, aaO. (FN 22), S. 381.

82 McCleskey v. Zant, 499 U.S. 467 (1991). Ebd.

83 Payne v. Tennessee, 501 U.S. 808 (1991). Ebd.

84 Roper v. Simmons, No. 03-633 (2005).

85 Charles Lane, "Justices Back Detainee Access To U.S. Courts. Presidents's Powers Are Limited « in: The Washington Post, 29. 06. 2004.

86 Rasul v. Bush, 03-334 (2004).

87 Todd S. Purdum, »In Classic Check and Balance, Court Shows Bush It Also Has Wartime Powers « in: The New York Times, 29. 06. 2004.

88 Hamdiv. Rumsfeld, 03-6696 (2004). 


\section{Schlussbetrachtung}

\subsection{Resümee}

Zu dem Zeitpunkt, als Earl Warren 1969 nach sechzehnjähriger Tätigkeit als Chief Justice des Supreme Court der Vereinigten Staaten sein Amt zur Verfügung stellte, gab es keinerlei Zweifel daran, dass das Gericht sich während seines Vorsitzes endgültig zu einer gleichberechtigten Gewalt neben der Exekutive und dem Kongress etabliert hatte. Diese Entwicklung war zu Beginn der Amtszeit Earl Warrens 1953 nicht absehbar gewesen ${ }^{89}$, auch wenn die Grundsteine dafür durch die sieben Richterernennungen F.D. Roosevelts schon vor längerer Zeit gelegt worden waren. Die vormals existente wirtschaftsliberale laissez-faire Ausrichtung, die das Gericht über Jahrzehnte geprägt hatte und die jeglicher Ausweitung bundesstaatlicher Kompetenz in der allgemeinen Sozial- und Wirtschaftsgesetzgebung entgegenstand, war in ihrer Mehrheitlichkeit aufgebrochen worden. Die Entscheidungen des Warren Courts, die überwiegend liberaler Natur waren, stießen sowohl in der Administration Eisenhowers als auch in großen Teilen der Bevölkerung auf Unverständnis und heftige Gegenwehr. Erst das politische Klima der Regierungen unter Kennedy und Johnson bot dem Gericht die Möglichkeit verstärkter Unterstützung und wachsender Akzeptanz seiner Urteile. Zudem gelangte das Gericht Anfang der 60er Jahre durch die Ernennung Goldbergs an die notwendige liberale swing vote ${ }^{90}$, welche die Mehrheitsfindung innerhalb des Gerichts zugunsten der progressiven Rechtsprechung erleichterte. Diese sollte die Grundlage für die liberale Verfassungsinterpretation der letzten Jahre des Warren Courts sein. Der Burger Court wird aus der heutigen Perspektive gerne als transitional court, also als »Übergangsgericht « klassifiziert, wobei der Begriff den vielen wichtigen Entscheidungen, die durch dieses Gericht getroffen wurden, nicht gerecht wird. Das Gericht knüpfte auch unter dem Vorsitz von Warren E. Burger in vielen Verfassungsfragen an die Rechtsprechung des Warren Court an. Zwar dominierten, vor allem gegen Ende seiner Ära, die restriktiven Urteile, dennoch traf er anfangs bei Themen, die zu Zeiten des Warren Court nicht oder nur marginal verhandelt wurden, durchaus liberale Entscheidungen ${ }^{91}$. Mit zunehmender Besetzung durch moderate und konservative Richter ging dieser Trend jedoch zusehends zurück. Als schließlich 1986 William H. Rehn-

89 Earl Warren war vor Beginn seiner Tätigkeit ein durchwegs erfolgreicher republikanischer Politiker gewesen, der in den frühen Jahren seiner Amtszeit als Gouverneur von Kalifornien (1943-53) konservative Ansichten vertrat. Zum Beispiel unterstützte er während des 2. Weltkrieges die Verfügung, alle Bewohner der Westküste, die japanischer Abstammung waren, zu internieren. Nach dem Ende des 2. Weltkrieges wurde Warrens liberalpolitische Transformation langsam daran erkennbar, dass er zum Verfechter einer staatlich unterstützten Krankenversicherung und von sozialen Fürsorgeleistungen wurde. Kenneth Jost, »Earl Warren« in: Jost, The Supreme Court A to Z, aaO. (FN 32), S. 505-506.

90 Vgl. Powe Jr., The Warren Court and American Politics, aaO. (FN 12), S. 211.

91 Zum Beispiel die Urteile Furman v. Georgia, 408 U.S. 238 (1972) und Roe v. Wade, 410 U.S. 113 (1973). 
quist von Präsident Reagan zum Chief Justice ernannt wurde, war die Entwicklung zu einem verstärkt an konservativen Richtlinien orientierten Supreme Court bereits weit fortgeschritten ${ }^{92}$. Die Tatsache, dass die Richterernennung über fast drei Jahrzehnte allein in der Hand von republikanischen Präsidenten lag, forcierte diesen Prozess. In den vorhergegangenen Kapiteln wurde deutlich, dass mit Beginn der 70er Jahre in den behandelten Verfassungsfragen die eine oder andere "Trendwende«stattfand. Beeinflusst durch die konservative Politik der Exekutive, verabschiedete sich das Gericht nach und nach vom dem liberalen judicial activism der 50er und 60er Jahre. Dies betrifft besonders die Rechtsprechung in den Bereichen der Desegregation von Schulen, die Haltung gegenüber affirmative action-Programmen, die Auslegung der Persönlichkeitsrechte und nicht zuletzt die Interpretation der bei der Strafverfolgung relevanten Zusatzartikel und die Bestrafung von Tätern beziehungsweise des Strafmaßes. Während die Richter der Burger und Rehnquist Courts die vom Warren Court initiierten "Verfassungsreformen" weitgehend respektierten, weiteten sie diese Reformen weder aus, noch lehnten sie ihre eigene Rechtsprechung direkt an die des liberalen Warren Courts an. Vielmehr praktizierten die Richter eine Jurisdiktion, die in vielen Aspekten moderate, in der RehnquistÄra dann verstärkt konservative Grundzüge aufweist. Zwangsläufig hat diese Art der Rechtsprechung auf Dauer Änderungen im Bereich der Verfassungsinterpretation nach sich gezogen. So ist das von konservativer Seite stark bekämpfte busing heute vollständig von der Agenda des Supreme Courts verschwunden ${ }^{93}$. Auch die Politik der Minderheitenförderung wurde durch den Rebnquist Court deutlich eingeschränkt, mit der Konsequenz, dass Maßnahmen in diesem Bereich seit Jahren stark rückläufig sind. Die erneute Bestätigung des Bakke Urteils Ende Juni 2003 bekräftigte dies erneut, auch wenn die Politik der Minderheitenförderung dank des Grutter Urteils vorerst ${ }^{94}$. Doch gilt es dabei erneut zu betonen, wie knapp das Urteil (5-4 Stimmen) zustande kam. Noch sind die Konsequenzen, welche die knappe

92 Den nachstehenden Slogan aus seinem 1980er Wahlkampf hatte Reagan zu dem damaligen Zeitpunkt schon mit der Ernennung der Richterin Sandra Day O'Connor und dem Richter Antonio Scalia zu großen Teilen eingelöst: »So my agenda is [...] to appoint judges [...] who don't invent new or fanciful constitutional rights [...], judges who believe the courts should interpret the law, not make it; judges in short, who understand the principle of judicial restraint " Ronald Reagan, zitiert nach: Cornelia B. Riess, Reagans Richter: Der Supreme Court in der Innenpolitik der 80er Jabre, Frankfurt 1993, S. 11.

93 Die busing Maßnahmen scheiterten aus mehreren Gründen. Zum einen zogen viele weiße Familien, die es sich leisten konnten, in die Vorstädte, um den Integrationsmaßnahmen zu entfliehen. Was wiederum zu einem enormen Ungleichgewicht zwischen innerstädtischen und vorstädtischen Schulen geführt hat. Und zwar nicht nur bezüglich der Zusammensetzung nach Hautfarbe, sondern auch bezüglich der Finanzmittel, die den Schulen zur Verfügung stehen.

94 Wie bereits eingangs erwähnt unterstützte die Bush Administration die zwei weißen Klägerinnen, die mit ihrer Anfechtung der affirmative action Programme der Universität von Michigan zwar einen der beiden Fälle gewannen, aber mit ihrem Hauptziel, nämlich der endgültigen Abschaffung solcher Programme, scheiterten da der zweite Fall zugunsten der Universität entschieden wurde. Vgl. Greg Winter, »Ruling Provides Relief, But Less Than Hoped « in: The New York Times, 24. 06. 2003. 
Entscheidung für die Motivation der affirmative action-Gegner haben kann, nicht absehbar. Jedoch könnte eine wachsende Motivation, affirmative action erneut anzufechten, besonders dann weiter ansteigen, wenn ein Richter (vornehmlich einer aus dem moderaten oder liberalen Lager) altersbedingt sein Amt zur Verfügung stellt. Genau dieser Fall ist durch den Rücktritt der Richterin O'Connor, die 2003 durch ihre Stimme die (vorläufige) Aufrechterhaltung der Minderheitenförderung möglich machte, eingetreten. Präsident Bush hat nunmehr zum ersten Mal in seiner Amtszeit die Chance einen Richter an den Obersten Gerichtshof zu berufen. Diese Ernennung, übrigens die erste seit elf Jahren, wird mit hoher Wahrscheinlichkeit nicht die einzige bleiben. Zwei der verbleibenden acht Richter sind über achtzig Jahre alt, einer der beiden, Chief Justice Rehnquist leidet an Schilddrüsenkrebs und hat wegen seiner Behandlung in der letzten Sitzungsperiode an einigen Verhandlungen nicht teilnehmen können. Obwohl der vorsitzende Richter jegliche Rücktrittsgerüchte verneint, ist es sehr fraglich, dass er die zweite Amtszeit Bushs in höchstrichterlichen Würden überdauern wird. Also ist davon auszugehen, dass George W. Bush noch mindestens einen weiteren Kandidaten an das Gericht berufen darf. Damit bietet sich für ihn die Chance, das bisher in vielen Bereichen doch eher moderat konservative Gesicht des Supreme Court nachhaltig verändern zu können. Besonders dann, wenn die abdankenden Richter dem liberalen oder moderaten Flügel des Supreme Court zuzurechnen sind, kann eine Verschiebung der Mehrheiten hin zu einer weniger dynamischen, wenn nicht sogar regressiven Verfassungsauslegung erfolgen, die nicht nur die Zukunft der Minderheitenförderung in Frage stellen würde.

Weiterhin nahm das Gericht unter Warren E. Burger und William H. Rehnquist deutlich Abstand von der weiten Definition der Privatsphäre95, wie sie einst der Warren Court vertrat, so zum Beispiel in Belangen der Abtreibung und der Homosexualität. Auch wenn Chief Justice Rehnquist sein erklärtes Ziel, die Entscheidung Roe v. Wade zu kippen, bisher noch nicht umsetzen konnte, so fand unter seinem Vorsitz doch eine deutliche Beschneidung des Rechts auf Abtreibung statt. Darüber hinaus setzte sich auch in der Thematik der Strafverfolgung und im Bereich der Todesstrafe eine deutlich konservativere Verfassungsinterpretation durch. Dabei weisen gerade diese beiden Themen enorme prozessuale Defizite auf, einerseits wegen der nachweislichen Voreingenommenheit gegenüber Straftätern, die Minderheiten angehören ${ }^{96}$, andererseits aufgrund der inhärenten Systemdefizite ${ }^{97}$, welche besonders im Bereich des capital punishment zum Tragen kommen. Eine Abschaffung der Todesstrafe insgesamt steht aufgrund der momentanen Konstellation des Gerichts

95 Die einzige Ausnahme bildet der oben thematisierte Fall Lawrence v. Texas, der ebenfalls Ende Juni $2003 \mathrm{zu}$ seinem abschließenden Urteil gekommen ist. Allerdings trat in diesem Fall die ideologische Spaltung des Gerichts über bestimmte Grundrechtsfragen besonders deutlich zu Tage (man möge nur die Mehrheitsmeinung des Gerichts mit dem Dissent des Richters Scalia vergleichen). Ebd.

96 Jürgen Martschukat, Die Geschichte der Todesstrafe in Nordamerika, München 2002, S. 179.

97 Ebd., S. 180-184. 
in naher Zukunft wohl eher nicht bevor ${ }^{98}$. Weiterhin vollzog das Gericht eine grundlegende Neuordnung im Bereich der Machtverteilung zwischen Bundesstaat und Einzelstaaten. War das Gericht seit der New Deal-Ära großzügig mit der Gewährung bundesstaatlicher Kompetenzen gewesen, kehrte der Rebnquist Court diesen Trend zugunsten der Einzelstaaten wieder um ${ }^{99}$. Zwar sollte sich das positiv auf die Entwicklung der Eigentumsrechte und der unternehmerischen Freiheit auswirken, hatte gleichzeitig aber gravierende Auswirkungen auf das Sozialsystem ${ }^{100}$, die Neueinteilung von Wahlkreisen und die Verbindlichkeit der Bill of Rights für die Einzelstaaten. Es gibt aber auch Bereiche, in denen die Rechtsprechung über Jahre hinweg weitgehende Konstanz aufweist. Die Rede- und Ausdrucksfreiheit wurde unter dem Burger Court stark ausgeweitet, so fiel zum Beispiel auch die kommerzielle Rede unter den Schutz des 1. Zusatzartikels. Zwar wurde diese Art der Rede bereits im Rehnquist Court wieder strikter ausgelegt, das flag burning-Urteil des Gerichts macht die Bedeutung, die dem 1. Zusatzartikel auch weiterhin zugemessen wurde, deutlich ${ }^{101}$. Man muss in diesem Fall aber berücksichtigen, dass Johnson nur dank einer knappen 5-4 Entscheidung freigesprochen wurde und im Zuge der öffentlichen Empörung vier legislative Anläufe genommen wurden, um das Urteil mittels eines Verfassungszusatzes zu revidieren, die jedoch allesamt am Senat scheiterten ${ }^{102}$. Zudem zeichnete sich in den letzten Jahren ab, dass das Gericht langsam Abstand von der strikten Trennung zwischen Religion und Staat nimmt. Das Tempo dieser Entwicklung und die durch die knappen Mehrheitsverhältnisse verursachten Inkonsistenz der Rechtssprechung, die erst vor kurzem wieder zu Tage trat ${ }^{103}$, verärgert vor allem die religiöse Rechte, die sich von einer Neuausrichtung des Gerichts besonders viel verspricht $t^{104}$.

98 Bei dieser Frage ist die persönliche Einstellung jedes einzelnen Richters ausschlaggebend, nicht seine oder ihre Parteinähe. Allgemein lassen sich die Befürworter der Todesstrafe in den Vereinigten Staaten nicht auf konservative Richter und Politiker beschränken. In allen politischen Lagern gibt es Verfechter dieses »Strafmaßes«, generell unterstützt eine deutliche Mehrheit der amerikanischen Bevölkerung die Todesstrafe.

99 Vgl. Kelly, The American Constitution, aaO. (FN 19), S. 757.

100 Vgl. Edward Lazarus, »Assessing Rehnquist's Legacy In The Final Chapter Of His Time As Chief Justice" in: Find Law's. Legal Commentary, 26. 12. 2000, http:// writ.news.findlaw.com/lazarus/20001226.html (abgerufen am 18. 02. 2003.)

101 Texas v. Johnson, 491 U.S. 397 (1989). Johnson war unter einem Gesetz des Bundesstaates Texas angeklagt worden, da er während einer Demonstration die amerikanische Flagge verbrannt hatte. Anhand dieses Statuts wurde er zu einer Freiheitsstrafe von einem Jahr Gefängnis und einer Geldstrafe von \$ 2000 verurteilt. Goldman, Constitutional Law, aaO. (FN 26), S. 410-460, S. 455-460.

102 Robert A. Rosenbaum / Douglas Brinkley (Hg.), The Penguin Encyclopedia of American History, New York u. a. 2003, S. 126.

103 Ein Urteil des Gerichts Ende Juni dieses Jahres hielt das Aufstellen eines Monuments mit den Zehn Geboten vor dem Sitz des texanischen Parlaments für verfassungskonform (Van Orden v. Perry, No. 03-1500 (2005)), ein anderes wiederum verbat die Ausstellung dieser, in Posterform, in zwei Gerichtsgebäuden in Kentucky (McCreary v. American Civil Liberties Union of Kentucky, No. 03-1693 (2005)).

104 Neben der Abschaffung des Rechts auf Abtreibung, die Wiedereinführung des Schulgebets, der Beibehaltung der Wörter »unter Gott « im Pledge of Allegiance, das Ende der Minderheitenförderung und die Verhinderung der Ehe für gleichgeschlechtliche Paare usw. 


\subsection{Ausblick}

Der Trend in vielen verfassungsrechtlichen Themen ging in den letzten Jahrzehnten verstärkt in die Richtung einer engen, verstärkt an konservativen Werten orientierten Verfassungsauslegung. Dennoch entsprach das Gericht bis heute nie ganz den Erwartungen konservativer Politiker. Diese forderten paradoxerweise die richterliche Zurückhaltung, erwarteten aber gleichzeitig von dem Gericht, dass bestimmte Präzedenzfälle ${ }^{105}$ revidiert werden, was sich wiederum nur durch richterlichen Aktivismus bewerkstelligen ließe.

Schon zu Gründungszeiten der Vereinigten Staaten betonte Alexander Hamilton die bedeutende Rolle, die das Gericht bezüglich des Schutzes von Minderheiten innehat. Dabei sah er die richterliche Unabhängigkeit als Voraussetzung dafür an, dieser Pflicht gerecht zu werden ${ }^{106}$. Jedoch nahm der Druck, den die Exekutive auf die Richter ausübt, besonders seit den 70er Jahren des 20. Jahrhunderts, stetig zu. Die Forderung, dass den Wünschen der Administration und der Mehrheit immer entsprochen wird, mag für diese zwar verlockend sein, sie ist mit dem Anspruch eines Rechtsstaates aber unvereinbar. Die Hauptaufgabe des Gerichtes, namentlich die Überprüfung von Gesetzen und Rechtsverordnungen auf ihre Verfassungsmäßigkeit, beugt der Gefahr vor, dass Minderheiten auf der Strecke bleiben, die wenig Gewicht im politischen Prozess eines Majoritätssystems besitzen. Außerdem würde eine solche »Gleichschaltung « dem System der »checks and balances" zuwiderlaufen.

Gerade heute aber ist die politische Unabhängigkeit des Obersten Bundesgerichts gefragt. Die Zäsur im amerikanischen Rechtssystem, die nach dem 11. September 2001 stattgefunden hat, verlangt danach. Es wird jedoch außerhalb des Machtbereichs des Supreme Court liegen, die geplanten Zusätze zur Notstandsgesetzgebung ${ }^{107}$ auf ihre Verfassungsmäßigkeit zu überprüfen, sollten diese tatsächlich die legislative Hürde überwinden, da die genannten Gesetze gegen jegliche Form des judicial review "geschützt« wurden. Dies wäre ein Novum in der amerikanischen Rechtsgeschichte und würde dem Supreme Court das nötige Instrumentarium entziehen, um seiner Rolle als »Hüter « der Verfassung gerecht zu werden. Gerade in Krisenzeiten, in welchen die Abwägung zwischen nationaler Sicherheit und individuellen Freiheitsrechten oft zur Einschränkung letzterer führen, ist die Kompetenz des Gerichts unentbehrlich.

105 Beispielsweise das Recht auf Abtreibung und die Politik der Minderheitenförderung.

106 Vgl. Hamilton, Alexander, »78. Artikel. Zur Gestaltung der rechtsprechenden Gewalt, insbesondere der Amtsdauer auf Lebenszeit« in: Angela Adams / Willi Paul Adams, Hamilton/Madison/Jay, Die Federalist Artikel. Politische Theorie und Verfassungskommentar der amerikanischen Gründerväter, Paderborn u. a. 1994, S. 469-477, S. 475.

107 Patriot Act; sowie den Entwurf des Domestic Security Enhancement Act (auch bekannt unter dem Namen Patriot II), welcher Anfang 2003 ohne Wissen des Justizministeriums (und folglich ohne dessen Einverständnis) veröffentlicht wurde. Vgl. Anita Ramasatry, "Patriot II: The Sequel Why It Is Even Scarier Than The First Patriot Act in: Find Law's. Legal Commentary, 7.02.2003, http://writ.news.findlaw.com/scripts/ printer_friendly.pl?page $=/$ ramasastry/20030217.html (abgerufen am 28. 02. 2003). 
Einige Urteile der letzten Jahre haben auf konservativer Seite großen Unmut erzeugt, - dieser äußerte sich in letzter Zeit vermehrt in Gesetzesinitiativen von Kongressmitgliedern, die das Ziel haben die richterliche Unabhängigkeit zu beschränken. Der vorsitzende Richter, wahrlich nicht im Verdacht ein Liberaler zu sein, drückte deutlich seinen Unmut über diese Vorgehensweise der Legislative aus ${ }^{108}$. $\mathrm{Ob}$ diese Attacken gegen die Judikative nach der Neuausrichtung des Gerichts durch Präsident Bush aufhören, bleibt abzuwarten. Fest steht, dass in diesem Sommer Bewegung in die amerikanische Innenpolitik kommt.

\section{Zusammenfassung}

Alexander Hamilton bezeichnete die Judikative als die »least dangerous branch « der drei Gewalten im politischen System der USA. Dennoch hat sich der Supreme Court innerhalb der letzten 200 Jahre zu einer veritablen Machtinstanz entwickelt, welche der Exekutive und der Legislative nicht selten das Leben schwer machte. In den zurückliegenden sechs Jahrzehnten musste sich das Gericht des Öfteren mit Verfassungsstreitigkeiten wie der Desegregation, der Minderheitenförderung, der Abtreibung und strafrechtlichen Verfassungsfragen auseinandersetzen. Die vom Gericht gefällten Urteile waren hierbei sehr unterschiedlich. Faktoren wie die Parteizugehörigkeit und die ideologische Einstellung der Richter spielen bei der Beschäftigung mit Verfassungskontroversen eine wachsende Rolle. Mancher Präsident nahm harte Nominierungskämpfe in Kauf, um die Politik bis weit nach dem Ende seiner Amtszeit zu beeinflussen. Dieser Versuch einer konservativen Wende beim Obersten Gerichtshof hat bei der Verfassungsrechtsprechung merkliche Spuren hinterlassen.

\section{Summary}

Alexander Hamilton argued that the federal judiciary will always be the »least dangerous branch to the political rights of the Constitution «. Hamilton's assessment of the need of a federal Supreme Court was accurate, but he underestimated its importance. In its more than 200 years of existence the justices of the Court have made the job of the executive and the legislative branches sometimes quite difficult. During the last six decades, the Court has several times dealt with constitutional questions concerning desegregation, affirmative action, equal protection of the law, abortion and criminal procedure. Party membership and ideological attitudes have become increasingly important when certain controversial cases are heard in the court. In the past and present, presidents have put up with harsh nomination battles in order to ascertain that their political influence will prevail far beyond their office terms. This attempt of a conservative redefinition has had decisive impact on the American constitutional interpretation.

108 Vgl. William H. Rehnquist, »2004 Chief Justice’s Year-End Report On The Federal Judiciary « http://www.supremecourtus.gov/publicinfo/year-end/2004year-endreport.pdf (abgerufen am 20. 04. 2005). 